Chirurg 2016 $87: 251$

DOI 10.1007/s00104-016-0165-6

Online publiziert: 24. Februar 2016

๑) Springer-Verlag Berlin Heidelberg 2016

CrossMark

\section{Anthuber - M. Schrempf}

Klinik für Allgemein-, Viszeral- Transplantationschirurgie, Klinikum Augsburg, Augsburg, Deutschland

\title{
Metaanalyse zur perioperativen Antibiotikagabe im Rahmen der laparoskopischen Cholezystektomie
}

\section{Originalpublikation}

Pasquali S, Boal M, Griffiths EA et al (2016) Meta-analysis of perioperative antibiotics in patients undergoing laparoscopic cholecystectomy. Br J Surg 103:27-34. doi: $10.1002 /$ bjs. 9904

Hintergrund. Die laparoskopische Cholezystektomie (CHE) zählt weltweit zu den häufigsten viszeralchirurgischen Eingriffen. Bei Patienten, die eine laparoskopische CHE aufgrund einer symptomatischen Cholezystolithiasis oder leicht- bis mittelgradigen Cholezystitis erhalten, ist die Effektivität einer perioperativen Antibiotikagabe mit dem Ziel, Infektionen im Operationsfeld (Surgical Site Infection; SSI) oder nosokomiale Infektionen $\mathrm{zu}$ reduzieren, unklar. Gleichzeitig wachsen die Bestrebungen, den unnötigen Einsatz von Antibiotika in der Medizin zu reduzieren, um der zunehmenden Entwicklung von Antibiotikaresistenzen und der steigenden Anzahl an Clostridium-difficile-Infektionen entgegenzuwirken.

Methoden. Zur Suche nach geeigneten Artikeln wurden die MEDLINE-, Embase- und Cochrane-Datenbanken verwendet. Es wurden ausschließlich randomisierte kontrollierte Studien (RCT) in englischer Sprache eingeschlossen. Kriterium für den Einschluss war der Vergleich einer perioperativen Antibiotikagabe (prä- und/oder postoperativ) mit einer Placebogabe oder keiner Antibiotikagabe im Rahmen einer laparoskopischen CHE, bei symptomatischer Cho- lezystolithiasis oder leicht- bis mittelgradiger Cholezystitis. Ausgewertet wurden unter anderem die Häufigkeit an SSIs sowie die Gesamtzahl an nosokomialen Infektionen (einschließlich SSIs) innerhalb von 30 Tagen nach laparoskopischer CHE. Die Analyse erfolgte anhand eines Random-Effects-Modells.

Ergebnisse. 19 RCTs aus den Jahren 1997 bis 2015 mit einer Gesamtzahl von 5259 Teilnehmern wurden in die Metaanalyse eingeschlossen. 2709 (51,5\%) Teilnehmer wurden mit einem Antibiotikum behandelt. Die Häufigkeit von SSIs und nosokomialen Infektionen betrug 2,4\% bzw. 4,2\% unter den Patienten, die eine perioperative Antibiotikagabe erhielten, und 3,2\% bzw. 7,2\% unter den Patienten, die kein Antibiotikum erhielten. Eine Antibiotikagabe führte weder zur signifikanten Reduktion der SSIs (relatives Risiko (RR) 0,81; $95 \%$-Konfidenzintervall [KI] $0,58-1,13 ; p=0,21)$ noch der nosokomialen Infektionen (RR 0,64; $95 \%$-KI $0,36-1,14 ; p=0,13)$. Für SSIs bestand keine signifikante Heterogenität zwischen den Studien ( $n=19)$, allerdings war für nosokomiale Infektionen zwischen den Studien $(n=8)$ eine signifikante Heterogenität nachweisbar. Eine Subgruppenanalyse aus Studien mit ausschließlich präoperativer Antibiotikagabe $(n=14)$ konnte ebenfalls keine signifikante Reduktion an SSIs nachweisen (RR 0,91; $95 \%$-KI $0,60-1,39 ; p=0,66)$.

Diskussion und Beurteilung. Die vorliegende Metaanalyse zeigt, dass eine perioperative Antibiotikagabe im Rahmen der laparoskopischen CHE bei symptomatischer Cholezystolithiasis und leicht- bis mittelgradiger Cholezystitis die Anzahl an SSIs und nosokomialen Infektionen nicht reduziert. Dieses Ergebnis wird durch zwei große populationsbasierte Studien gestützt. Die schwedische „Registry of Gallstone Surgery and ERCP“ fand bei der Auswertung von 13.911 Patienten mit notfallmäßiger CHE [1] und 10.927 Patienten mit elektiver CHE [2] keinen Vorteil für eine Antibiotikagabe. Diese Ergebnisse sollten unverzüglich Eingang in die klinische Routine auch in Deutschland finden, um unnötige Antibiotikagaben und deren Folgen zu reduzieren.

\section{Korrespondenzadresse}

\section{Prof. Dr. M. Anthuber}

Klinik für Allgemein-, Viszeral- Transplantationschirurgie, Klinikum Augsburg Stenglinstr. 2, 86156 Augsburg, Deutschland matthias.anthuber@klinikum-augsburg.de

Interessenkonflikt. M. Anthuber und M. Schrempf geben an, dass kein Interessenkonflikt besteht.

\section{Literatur}

1. Jaafar G, Persson G, Svennblad B, Sandblom G (2014) Outcomes of antibiotic prophylaxis in acute cholecystectomy in a population-based gallstone surgery registry. Br J Surg 101:69-73

2. Lundström P, Sandblom G, Osterberg J et al (2010) Effectiveness of prophylactic antibiotics in a population-based cohort of patients undergoing planned cholecystectomy. J Gastrointest Surg 14:329-334 